\title{
PRIMER EPISODIO PSICÓTICO: ABORDAJE DE ENFERMERÍA DESDE UNA UNIDAD DE ADOLESCENTES
}

\section{MARÍA JOSEFA MARTÍNEZ CARREÑO}

Enfermera asistencial. Departamento de Psiquiatría.

Hospital General Universitario Gregorio Marañón. Madrid.

\section{INTRODUCCIÓN}

Alrededor del $3 \%$ de la población sufre un episodio psicótico a lo largo de su vida. El primer episodio psicótico (PEP) se da generalmente entre los 15 y los 30 años ${ }^{1}$.

Los datos epidemiológicos de los trastornos psicóticos en niños y adolescentes son muy limitados. El National Institute for Health and Care Excellence estima que la prevalencia de los trastornos psicóticos en niños y adolescentes de edad comprendida entre 5 y 18 años es del 0,4\% (prevalencia media poblacional en el Reino Unido: 0,7\%). El mayor aumento de la incidencia aparece a partir de los 15 años de edad².

\section{PRESENTACIÓN DEL CASO}

Paciente varón de 17 años, natural de Bolivia, derivado del Hospital La Paz (Madrid) para ingreso en Unidad de Adolescentes (UADO) para filiación de comportamiento. Niega consumo de tóxicos y no tiene antecedentes psiquiátricos de interés. Presentó

Correspondencia: M. J. Martínez

Correo electrónico: maria.jose.martinez.c@gmail.com una leucemia aguda mieloblástica M2 de alto riesgo en 2010 con trasplante alogénico en 2013, actualmente en remisión, y diabetes mellitus de tipo 2 , secundaria al tratamiento oncológico. De su infancia únicamente destaca que no mostraba actitud proactiva relacional, sin otras incidencias que reseñar. A primeros de abril de 2015 presenta comportamiento desorganizado, risas inadecuadas, verbaliza sensaciones extrañas, alucinaciones cenestésicas de tipo: «calor... que tengo que ir al infierno... noto que me agarran»; capacidad de poder cambiar aspectos físicos de su madre. Al ingreso presenta moderada angustia psicótica y bloqueos del pensamiento, enlentecimiento psicomotor, con tendencia a la minimización y ocultación de sintomatología delirante. Al avanzar el ingreso predomina la sintomatología negativa (aplanamiento afectivo y embotamiento emocional, apatía y abulia) y se niega al tratamiento farmacológico y a las ingestas, por lo que se llega a prescribir tratamiento por vía intramuscular y sondaje nasogástrico.

\section{VALORACIÓN DE ENFERMERÍA}

Se lleva a cabo la valoración a través de los patrones funcionales de Marjory Gordon ${ }^{3}$ y criterios de valoración enfermera estandarizada (variables) ${ }^{4}$ (tabla 1 ). 


\section{Percepción - manejo de la salud}

Eliminación

Actividad - ejercicio

Sueño - descanso

Cognitivo - perceptivo

Autopercepción autoconcepto

Rol - relaciones

Sexualidad reproducción

Tolerancia al estrés
(2908) Actitud ante su enfermedad: minimización de sintomatología. (2916) Actitud ante el régimen terapéutico: negación a tratamiento oral. (4506) Existencia de hábitos tóxicos: niega y no se objetivan en analítica.

(0310) Dieta específica: diabética.

(0302) Valoración de la alimentación: se niega a la ingesta oral de líquidos y sólidos.

(0103) IMC: 20,9.

(0316) Alteración del peso: pérdida de 3 kg en 15 días.

(0326) Sonda nasogástrica: por riesgo de desnutrición por ayuno.

Dentro de la normalidad.

(3823) Estudiante: repite $4 .^{\circ}$ de ESO.

(1501) Actividades de ocio: le gusta jugar al baloncesto.

(1506) Incapaz de ocio habitual: enlentecimiento psicomotor y tendencia al aislamiento.

(1326) Insomnio.

(3607) Confusión: ideas delirantes místico-religiosas.

(3712) Alteraciones psíquicas: alucinaciones cenestésicas (quemazón en cuello) y auditivas en forma de mensajes de Dios (elegido por él para mostrarle el Apocalipsis).

(3511) Fobias, miedos: a padecer la enfermedad de su padre.

(3513) Ansiedad: ante el ingreso, separación de su familia y su propia enfermedad.

(3805) Convive con: madre, hermanos y abuela materna.

(1431) Pérdidas significativas: fallecimiento de padre en 2008 diagnosticado de esquizofrenia.

No desea hablar de ello.

(2806) Agentes estresantes: en Navidades de 2014 el paciente se entera del diagnóstico de su padre y de la forma de fallecimiento (suicidio).

(4518) Minimiza los síntomas: sin conciencia de enfermedad al ingreso.

(1608) Creencias religiosas familiares: abuela materna perteneciente a iglesia adventista y defensora del ayuno como forma de purificación.

(1615) Prácticas religiosas de riesgo para la salud de la persona: ayuno como sacrificio. (Manifiesta que ya hizo un día de ayuno durante la quimioterapia y por ello se curó.)

IMC: índice de masa corporal. 


\section{DIAGNÓSTICOS Y PLANIFICACIÓN}

Se utiliza la taxonomía NANDA (North American Nursing Diagnosis Association $)^{5}$ y la clasificación de diagnósticos por patrones, NOC (Nursing Outco- mes Classification) ${ }^{6}$ y NIC (Nursing Interventions

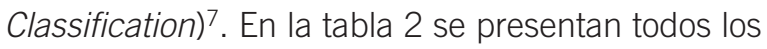
diagnósticos realizados y se desarrollan los relacionados más directamente con salud mental.

Tabla 2. Diagnósticos de enfermería y planificación

DG Incumplimiento 00079 r/c episodio psicótico m/p negativa a tomar medicación por vía oral.

DG Desequilibrio nutricional: ingesta inferior a las necesidades 00002 r/c ayuno voluntario m/p pérdida de peso y bajo tono muscular.

DG Riesgo de nivel de glucemia inestable 00179 r/c ayuno voluntario en paciente con DM2.

DG Riesgo de estreñimiento 00015 r/c ayuno voluntario.

DG Déficit de actividades recreativas 00097 r/c episodio psicótico m/p dejar de practicar deporte con los amigos, enlentecimiento psicomotor.

DG Déficit de autocuidado: alimentación 00102 r/c idea delirante de conseguir la salvación con el ayuno m/p negación a ingerir líquidos y/o sólidos.

DG Trastorno del patrón del sueño 00198 r/c ansiedad, ingreso y habitación compartida m/p despertares nocturnos y petición de medicación para conciliar el sueño.

DG Confusión aguda 00128 r/c brote psicótico m/p alteración en la percepción, actividad psicomotora y nivel de conciencia.

NOC

NIC
(0916) Nivel de delirio. Indicador:

- Alucinaciones

- Dificultad para interpretar estímulos del entorno

- Inquietud

(1403) Autocontrol del pensamiento distorsionado. Indicador:

- Reconoce que tiene alucinaciones o ideas delirantes

- Describe el contenido de las alucinaciones o ideas delirantes

(6440) Manejo del delirio. Actividades:

- Administrar medicación pautada.

- Monitorizar el estado neurológico de forma continua.

- Reconocer los miedos y sentimientos del paciente.

- No validar las percepciones incorrectas.

(6510) Manejo de las alucinaciones. Actividades:

- Establecer relación interpersonal de confianza.

- Administrar medicación pautada.

- Proporcionar al paciente la oportunidad de comentar las alucinaciones.

- Implicar al paciente en actividades que puedan distraerle de las alucinaciones.

(4820) Orientación de la realidad. Actividades:

- Presentar la realidad de manera que conserve la dignidad del paciente.

- Involucrar al paciente en actividades orientadas en la realidad.

- Animar a la familia a participar en los cuidados. 
DG Ansiedad 00146 r/c idea delirante de sufrir daños por demonios m/p sentimientos de aprensión, falta de concentración, insomnio, bloqueo del pensamiento e hiperatención.

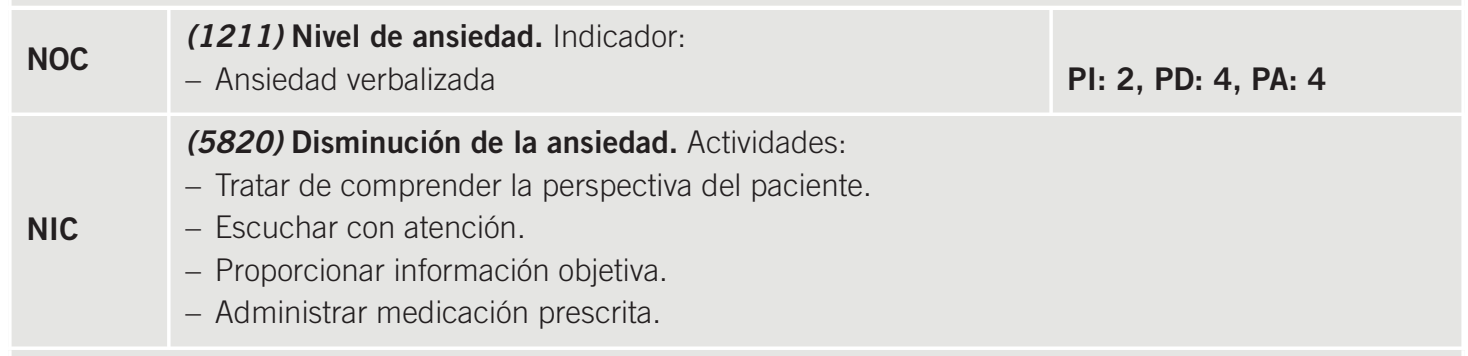

DG Temor 00148 r/c conocimiento de enfermedad paterna m/p verbalización de no querer padecer la enfermedad de su padre.
(1210) Nivel de miedo. Indicador:
- Preocupación por la fuente del miedo $\quad$ PI: 1, PD: 4, PA: 4

\section{(5230) Mejorar el afrontamiento. Actividades:}
NIC - Valorar la comprensión del paciente del proceso de enfermedad.
- Ayudar al paciente a clarificar conceptos equivocados y proporcionar información objetiva del diagnóstico, tratamiento y pronóstico.

DG Trastorno de la identidad personal 00121 r/c episodio psicótico m/p ideas de ser un elegido de Dios.

\begin{tabular}{l|l|l} 
NOC & (1215) Conciencia de uno mismo. Indicador: & \\
& - Mantiene la conciencia de los pensamientos & PI: 2, PD: 5, PA: 4 \\
\hline & (6450) Manejo de las ideas delirantes. Actividades: \\
NIC & - Establecer relación interpersonal de confianza. \\
& - Evitar reforzar ideas delirantes. \\
& - Tranquilizar al paciente sobre su seguridad. \\
& - Administrar tratamiento pautado.
\end{tabular}

DG Deterioro de la comunicación verbal 00051 r/c alteración de la percepción m/p dificultad para expresar los pensamientos verbalmente.

\begin{tabular}{l|l|l} 
NOC & (0903) Comunicación: expresiva. Indicador: & PI: 2, PD: 5, PA: 5 \\
& - Utiliza la conversación con claridad & (4920) Escucha activa. Actividades: \\
\hline & NIC & - Utilizar el silencia/escucha y preguntas y afirmaciones para animar a expresar pensamientos \\
& y preocupaciones. \\
& - Mostrar conciencia y sensibilidad hacia las emociones.
\end{tabular}


Tabla 2. Diagnósticos de enfermería y planificación (cont.)

DG Deterioro de la interacción social 00052 r/c episodio psicótico m/p tendencia al aislamiento, disconfort en las situaciones sociales.

\begin{tabular}{l|l|l} 
NOC & $\begin{array}{l}\text { (1502) Habilidades de interacción social. Indicador: } \\
- \text { Relaciones con los demás }\end{array}$ & PI: 2, PD: 4, PA: 4 \\
\hline & $\begin{array}{l}\text { (5100) Potenciación de la socialización. Actividades: } \\
\text { NIC }\end{array}$ & - Fomentar implicación en las relaciones ya establecidas. \\
& - Animar al paciente a desarrollar relaciones y proporcionar retroalimentación positiva cuando \\
& establezca contacto con los demás.
\end{tabular}

DG Negación ineficaz 00072 r/c miedo a padecer enfermedad mental m/p no pedir ayuda al comienzo de la sintomatología, no percibir la importancia de los síntomas o el riesgo personal y minimizar síntomas.

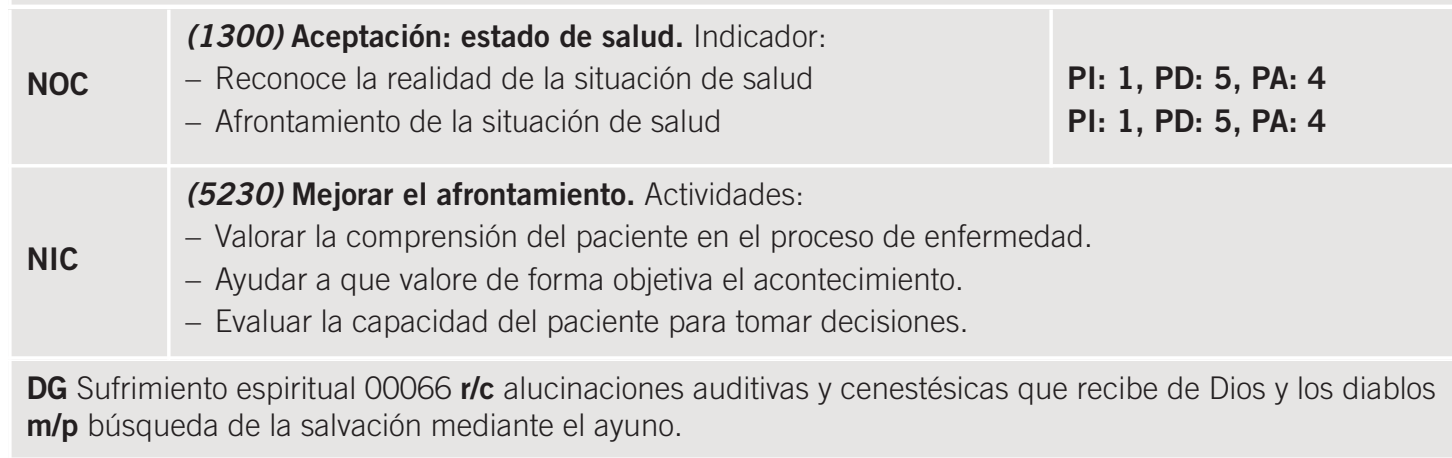

\begin{tabular}{|l|l|l|}
\hline NOC & $\begin{array}{l}\text { (1704) Creencias sobre la salud: percepción de amenaza. } \\
\text { Indicador: } \\
\text { - Experiencias místicas }\end{array}$ & PI: 1, PD: 5, PA: 4 \\
\hline NIC & (5420) Apoyo espiritual. Actividades: \\
\hline & - Estar abierto a las expresiones de preocupación del individuo. \\
& - Utilizar técnicas para ayudar al individuo a clarificar sus creencias. \\
& - Identificar conductas religiosas que potencien el delirio e informar a la familia.
\end{tabular}

DG: diagnóstico; m/p: manifestado por; PA: puntuación al alta; PD: puntuación deseada; PI: puntuación al ingreso; r/c: relacionado con. Escala de Likert: puntuación «5», la mejor posible; «1», la peor posible.

\section{EVALUACIÓN DEL PLAN DE CUIDADOS Y RESULTADOS}

Se plantea con este paciente seguir una línea terapéutica basada en tratamiento farmacológico para el control de la sintomatología psicótica, actividades grupales en terapia ocupacional para mejorar las relaciones interpersonales, y trabajo individualizado para tomar conciencia de su enfermedad. Se le pro- pone participar en un ensayo clínico en PEP con $\mathrm{N}$-acetilcisteína y en el Programa Piensa (Programa de Intervención en Psicosis Adolescente); tanto el paciente como su madre aceptan. Ambos programas se llevan a cabo a través del Servicio de Investigación del Servicio de Psiquiatría del niño y del adolescente ${ }^{8}$. Se procede al alta hospitalaria y seguimiento en el centro de salud mental de su área. 


\section{BIBLIOGRAFÍA}

1. Bernardo M, Bioque M, Parellada M, Saiz Ruiz J, Cuesta MJ, Llerena A, et al. Assessing clinical and functional outcomes in a gene--environment interaction study in first episode of psychosis (PEPs). Rev Psiquiatr Salud Ment. 2013;6(1):4-16.

2. Kendall T, Hollis C, Stafford M, Taylor C. Guideline Development Group. Recognition and management of psychosis and schizophrenia in children and young people: summary of NICE guidance. BMJ. 2013;346: f150.

3. Gordon M. Manual de diagnósticos de enfermería. 11. ${ }^{a}$ ed. Madrid: McGraw Hill/Interamericana; 2007.

4. Fuden, Observatorio de Metodología enfermera [citado el 20 de junio de 2015]. Disponible en: http://www. ome.es/04_01_desa.cfm?id=391\#ancla1
5. Herdman TH, editor. NANDA Internacional. Diagnósticos enfermeros. Definiciones y clasificación 20122014. Barcelona: Elsevier; 2012.

6. Moorhead S, Johnson M, Meridean ML, Swanson E, editores. Clasificación de resultados de enfermería (NOC). Medición de resultados en salud. 5. ${ }^{a}$ ed. Barcelona: Elsevier; 2013.

7. Bulechek GM, Butcher HK, Dochterman JM, Wagner $\mathrm{CM}$, editores. Clasificación de intervenciones de enfermería (NIC). 6. ${ }^{a}$ ed. Barcelona: Elsevier; 2013.

8. Servicio de Psiquiatría del niño y del Adolescente. Hospital General Universitario Gregorio Marañón [citado el 24 de junio de 2015]. Disponible en: http://www. madrid.org/cs/Satellite?cid=1142662634860\&language=es\&pagename=HospitalGregorioMaranon\%2FPag e\%2FHGMA_ServiciosPrincipal 